\title{
The Difference between the Two Representative Kampo Formulas for Treating Dysmenorrhea: An Observational Study
}

\author{
Tetsuhiro Yoshino, ${ }^{1}$ Kotoe Katayama, ${ }^{2}$ Yuko Horiba, ${ }^{1}$ Kaori Munakata, ${ }^{3}$ Rui Yamaguchi, \\ Seiya Imoto, ${ }^{4}$ Satoru Miyano, ${ }^{2}$ Hideki Mima, ${ }^{5}$ Kenji Watanabe, ${ }^{1,6}$ and Masaru Mimura ${ }^{7}$ \\ ${ }^{1}$ Center for Kampo Medicine, Keio University School of Medicine, 35 Shinanomachi, Shinjuku-ku, Tokyo 160-8582, Japan \\ ${ }^{2}$ Human Genome Center, The Institute of Medical Science, The University of Tokyo, 4-6-1 Shirokanedai, Minato-ku, \\ Tokyo 108-8639, Japan \\ ${ }^{3}$ SFC Laboratory, Keio University, 5322 Endo, Fujisawa, Kanagawa 252-0882, Japan \\ ${ }^{4}$ Division of Health Medical Data Science, Health Intelligence Center, The Institute of Medical Science, The University of Tokyo, \\ 4-6-1 Shirokanedai, Minato-ku, Tokyo 108-8639, Japan \\ ${ }^{5}$ School of Engineering, The University of Tokyo, 7-3-1 Hongo, Bunkyo-ku, Tokyo 113-8656, Japan \\ ${ }^{6}$ Faculty of Environment and Information Studies, Keio University, 5322 Endo, Fujisawa, Kanagawa 252-0882, Japan \\ ${ }^{7}$ Department of Neuropsychiatry, Keio University School of Medicine, 35 Shinanomachi, Shinjuku-ku, Tokyo 160-8582, Japan
}

Correspondence should be addressed to Kenji Watanabe; watanabekenji@keio.jp

Received 5 October 2015; Revised 19 January 2016; Accepted 27 January 2016

Academic Editor: Xingjiang Xiong

Copyright ( 92016 Tetsuhiro Yoshino et al. This is an open access article distributed under the Creative Commons Attribution License, which permits unrestricted use, distribution, and reproduction in any medium, provided the original work is properly cited.

In Kampo medicine, two different formulas are effective for treating dysmenorrhea-tokishakuyakusan and keishibukuryogan; however, the criteria by which specialists select the appropriate formula for each patient are not clear. We compared patients treated with tokishakuyakusan and those with keishibukuryogan and proposed a predictive model. The study included 168 primary and secondary dysmenorrhea patients who visited the Kampo Clinic at Keio University Hospital. We collected clinical data from 128 dysmenorrhea patients, compared the two patient groups and selected significantly different factors as potential predictors, and used logistic regression to establish a model. An external validation was performed using 40 dysmenorrhea patients. Lightheadedness, $\mathrm{BMI}<18.5$, and a weak abdomen were significantly more frequent in the tokishakuyakusan group; tendency to sweat, heat intolerance, leg numbness, a cold sensation in the lower back, a strong abdomen, and paraumbilical tenderness and resistance were more frequent in the keishibukuryogan group. The final model fitted the data well. Internally estimated accuracy was $81.2 \%$, and a leave-one-out cross-validation estimate of accuracy was $80.5 \%$. External validation accuracy was $85.0 \%$. We proposed a model for predicting the use of two Kampo formulas for dysmenorrhea, which should be validated in prospective trials.

\section{Introduction}

Dysmenorrhea is the most common gynecological disorder in women, regardless of age and nationality [1]. Patients with dysmenorrhea have strong lower abdominal or lower back pain that begins during or just before the menstrual period. Dysmenorrhea is thought to be caused by an excess or imbalance of prostanoids, and possibly other eicosanoids, released from the endometrium during menstruation. As a result, the uterine basal tone increases, with frequent and dysrhythmic contraction. Pain is induced by uterine hypercontractility, reduced uterine blood flow, and increased peripheral nerve hypersensitivity [2].

The standard treatment for dysmenorrhea is nonsteroidal anti-inflammatory drugs (NSAIDs) or oral contraceptives (OCs) $[3,4]$. Up to $30 \%$ of patients, however, do not respond sufficiently to NSAIDs, and $10 \%$ to $20 \%$ respond to neither NSAIDs nor OCs [1]. Furthermore, NSAIDs are contraindicated in patients with a peptic ulcer or gastritis. OCs are contraindicated in those with any thrombotic predisposing factor, breast cancer, migraine with aura, or pregnancy. For these reasons, various alternative treatments have been 
examined, such as acupressure, vitamin B1, vitamin E, use of a hot pack, transcutaneous electrical nerve stimulation, and behavioral interventions [5].

Kampo, Japanese traditional medicine, is a leading alternative medicine $[5,6]$ and is popular in Japan, particularly for treating women's health issues. Two Kampo formulas are commonly used for treating dysmenorrhea $[7,8]$ tokishakuyakusan and keishibukuryogan-and both have been shown to be effective in randomized placebo-controlled trials $[9,10]$. In the Japanese national health insurance system, both formulas are indicated for dysmenorrhea and other gynecological conditions, including irregular menstruation, menopause, and infertility.

Kampo formulas are prescribed according to traditional pattern-based diagnosis [11], which is used in addition to Western diagnosis [12]. In Kampo medicine, pattern diagnosis refers to the unique clinical classification of the patient, which takes into account symptoms, general constitution, and other factors. The patient is differentially diagnosed with chronic health conditions, including dysmenorrhea, on the basis of disharmony in any of the following areas: the eight categories (excess-deficiency, heat-cold, interiorexterior, and yin-yang) and body constituents (qi, blood, and fluid) [13]. Tokishakuyakusan is traditionally prescribed for patients diagnosed with "deficiency," "cold," "interior," "yin," "blood deficiency," and "fluid disturbance" [10], while keishibukuryogan is used for patients diagnosed with "excess," "tangled heat and cold," "interior," "yang," and "blood stasis."

However, pattern diagnosis in traditional medicine is a subtle art; it takes years to master the skills required to choose the appropriate formulas, and to our knowledge, it has not yet been reported whether the prescription of Kampo formulas by specialists can be predicted without knowledge of traditional pattern diagnosis. Moreover, it is not known how subjective symptoms and objective findings differ between patients who are prescribed the different Kampo formulas.

In this study, we compared the subjective symptoms and objective findings in patients prescribed tokishakuyakusan with those in patients prescribed keishibukuryogan and used this information to derive a model that can predict the selection of either of the two formulas by specialists in Kampo medicine.

\section{Methods}

2.1. Patient Enrollment. This observational study included primary and secondary dysmenorrhea patients who were first-time visitors to the Kampo Clinic at Keio University Hospital, between May 2008 and December 2015. All patients were treated with either of the two formulastokishakuyakusan or keishibukuryogan. Patients who were treated with both formulas were excluded. Patients over 50 years of age were also excluded. The Institutional Review Board at Keio University School of Medicine approved this study.

2.1.1. Comparison and Model-Development Analysis. In this analysis, we included patients who made their first visit between May 2008 and March 2013. Patients who were prescribed tokishakuyakusan were included in the "TSS" group, and those who were prescribed keishibukuryogan were in the "KBG" group. We used a browser-based questionnaire during this part of the study; the questionnaire is explained in detail in Section 2.2.

2.1.2. External Validation Analysis. The predictive model was validated using a different data set (the external validation group), obtained from patients who made their first visit to Kampo Clinic at Keio University Hospital between April 2013 and December 2015. We did not use the browser-based questionnaire system during this part of the study. The systems used in the medical interview were reviewed using a paperbased questionnaire, and this database was entirely separate from that used in the comparison and model-development analysis; however, the items in the questionnaire were identical.

2.2. Data Collection. In 2008, Keio University first introduced a browser-based questionnaire to collect information about patients' subjective symptoms, as well as their age, sex, body mass index (BMI), lifestyle, Western diagnosis (based on the international classification of diseases (ICD-10)), traditional medicine pattern-based diagnosis (based on ICD-11 beta version) [11], and Kampo formulas prescribed by Kampo specialists. Kampo specialists from representative Universities and Kampo institutions in Japan (Keio University, Chiba University, Toyama University, Jichi Medical University, Tokyo Women's Medical University, Tohoku University, Kameda Medical Center, and Aso Iizuka Hospital) prepared the questionnaire after repeated discussions. Using this questionnaire, which comprises 128 binary questions, we collected information about our patients' subjective symptoms, as described in our previous report [14].

BMI was assessed in 2 ways: as a sequential variable (crude BMI) and as binary variables: "slim" (yes/no) and "obese" (yes/no). Patients with a BMI $<18.5$ were considered slim, and those with a BMI $\geq 25$ were considered obese, as defined by the Japan Society for the Study of Obesity.

Data regarding each objective factor, including abdominal and tongue findings, were also collected as binary variables. Specifically, abdominal findings included nine items; one of these-abdominal strength-contained three mutually exclusive categories: weak, intermediate, and strong. Here, however, we used binary variables to code the abdominal strength: "weak abdomen" (yes/no) and "strong abdomen" (yes/no). Abdominal strength is determined by abdominal examination, whereby the doctor presses the palm of his/her hand onto the patients' abdomen to assess both the degree of resistance offered by the muscles and the thickness of the abdominal muscle wall and fat [15]. Other abdominal findings were also expressed in binary form, namely, epigastric discomfort, palpable abdominal aortic pulsation, hypochondrial resistance and discomfort, splashing sound in the epigastric region, paraumbilical tenderness and resistance, rectus muscle tension, weakness of the lower abdomen, and abdominal distension. Tongue findings included teeth marks on the edges of the patient's tongue and dilatation of the sublingual veins. 
2.3. Comparison of Tokishakuyakusan with Keishibukuryogan. We compared each subjective and objective item between the TSS and KBG groups. We used Fisher's exact test for comparison of binary variables and Wilcoxon's rank sum test and two-sample $t$-tests for continuous variables items, such as age and crude BMI. Missing data were ignored in the tests.

\subsection{A Predictive Model for Prescription of the Two Kampo Formulas by Specialists}

2.4.1. Selection of Potential Predictor Variables. We used variables with a $p$ value $<0.05$ in the analyses detailed in Section 2.3 as potential variables that could be used to predict which Kampo medicine would be prescribed. BMI had a $p$ value $<0.05$, but this information was missing for several patients; we therefore replaced the missing BMI data with the overall mean BMI during the model-development analysis.

2.4.2. Model-Fitting Procedure. We applied logistic regression to the 128 data points from the TSS and KBG groups [16]; the KBG group was designated as 1, and the TSS group as 0 . Using logistic regression analysis, we calculated the probability of the patient belonging to the KBG group; $p>$ 0.5 indicated that the patient was predicted to belong to the KBG group, and $p<0.5$ that the patient was predicted to belong to the TSS group. We then performed a univariate analysis on the potential predictive variables, followed by a multivariate analysis. The model that contained all the potential predictive variables was considered the full model. To measure the effect size of each predictive variable, we computed the odds ratio (OR).

However, to avoid overfitting the predictive model, the predictive variables needed to be selected more strictly, which we achieved using the Akaike information criterion (AIC) [17]. We started with the full model and challenged all possible models; the model with the lowest AIC was considered the final model.

The variance inflation factor (VIF) was used to monitor multicollinearity. We also evaluated interactions between predictor variables in the final model by including interaction terms along with main-effect terms. None of the interactions were found to be significant, and they are not discussed further in this paper.

2.5. Internal and External Validations of the Final Model. Calibration of the model was assessed using the area under the receiver operating characteristic curve (AUC) and the Hosmer-Lemeshow test [18]. An AUC $>0.80$ and a $p$ value $>0.05$ in the Hosmer-Lemeshow test were considered acceptable values. The final model was internally validated by leave-one-out cross-validation. We also externally validated the final model by applying it to the external validation group's data set.

2.6. Statistical Analyses. All statistical analyses were conducted using $\mathrm{R}$ software version 3.1.1 (The $\mathrm{R}$ Foundation for Statistical Computing; July 10, 2014; see also: http://www.r-project.org/). We used "glm" [19] from the package "stats," as well as the packages "DAAG" [20],
TABLE 1: Frequently used Kampo formulas in 222 patients with dysmenorrhea.

\begin{tabular}{lc}
\hline Formulas & Number \\
\hline Keishibukuryogan & 73 \\
Tokishakuyakusan & 67 \\
Kamishoyosan & 20 \\
Anchusan & 19 \\
Goreisan & 18 \\
Saikokeishikankyoto & 13 \\
Tokakujokito & 10 \\
Yokukansan & 8 \\
Saikokaryukotsuboreito & 8 \\
Tokikenchuto & 7 \\
Jumihaidokuto & 6 \\
Shosaikoto & 6 \\
Tokishigyakukagoshuyushokyoto & 6 \\
Bukuryoingohangekobokuto & 6 \\
Byakkokaninjinto & 6 \\
Daisaikoto & 5 \\
Hochuekkito & 5 \\
Shakuyakukanzoto & 5 \\
Hangekobokuto & 356 \\
Rikkunshito & 4 \\
Others (45 kinds of formulas) & 4 \\
\hline Total & 60 \\
\hline
\end{tabular}

Five patients from the keishibukuryogan group and 7 from the tokishakuyakusan group were excluded from the comparison and modeldevelopment analysis (see Figure 1). A total of 127 patients were prescribed 2 or more formulas, and 356 formulas were prescribed in total.

"pROC" [21], and "ResourceSelection" [22]. Data are shown as mean \pm standard deviation. We used a significance level of $5 \%$ for all tests but made no adjustment for multiple testing.

\section{Results}

3.1. Participant Information. We assessed the eligibility of 290 dysmenorrhea patients-222 patients for the comparison and model-development analysis and 68 patients for the external validation analysis.

Among the 222 candidate patients for the comparison and model-development analysis, 127 had been prescribed two or more formulas (a total of 356 formulas were prescribed; Table 1). Tokishakuyakusan and keishibukuryogan were the most frequently used formulas, and 135 patients (61\%) were prescribed either or both of these. None of the patients withdrew from the study. We excluded two patients who were aged over 50 years and six patients who were prescribed both tokishakuyakusan and keishibukuryogan or related formulas. Finally, we used data from 128 patients in the comparison and model-development analysis, comprising 60 who were prescribed only tokishakuyakusan (TSS group) and 68 who were prescribed only keishibukuryogan (KBG group; Figure 1: the comparison and model-development set).

Of the 68 candidate patients for the external validation analysis, 28 were excluded because they were not prescribed 


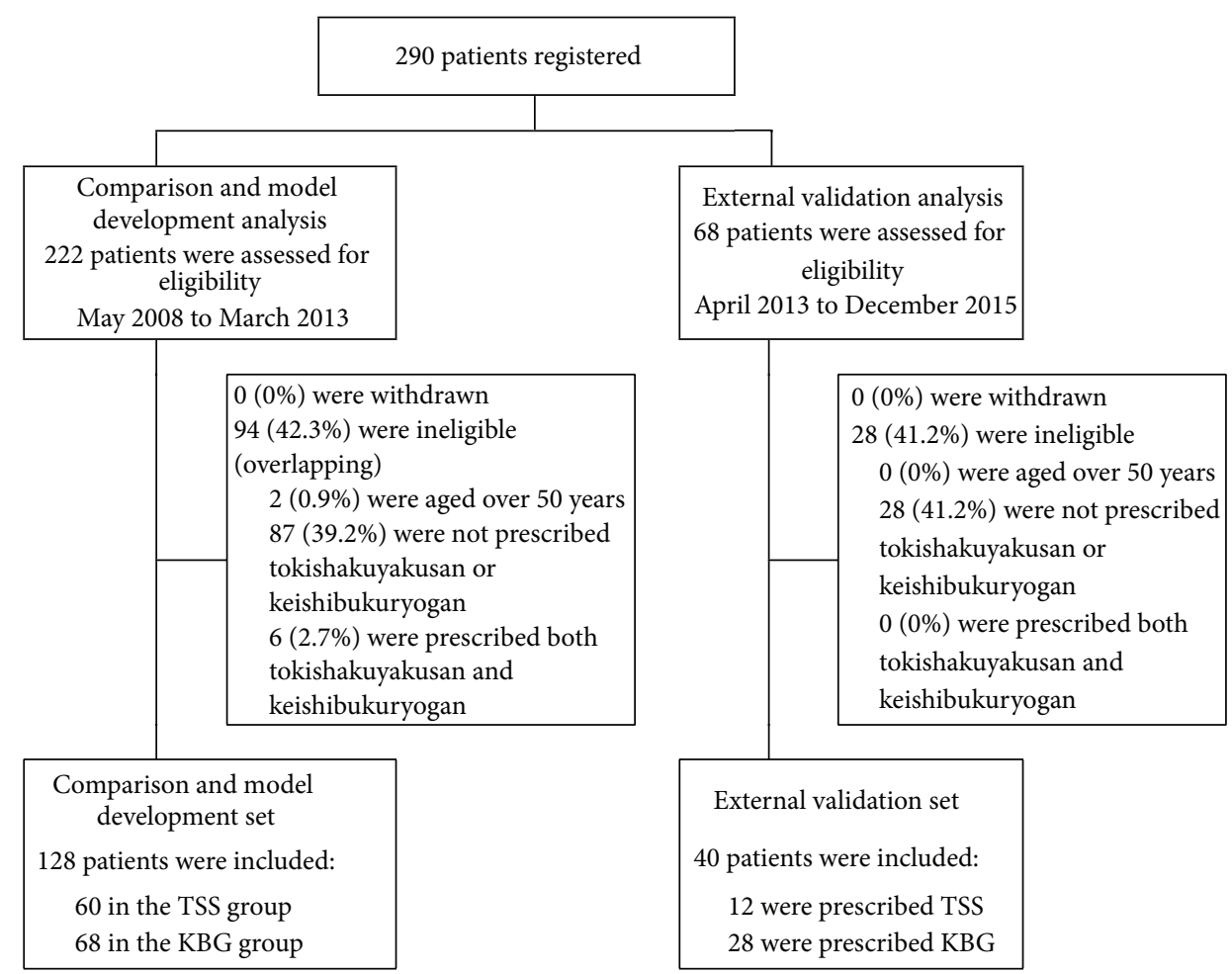

FIGURE 1: Patients' flow chart. Patients who were prescribed tokishakuyakusan only were included in the "TSS" group, and those who were prescribed keishibukuryogan only were included in the "KBG" group.

either tokishakuyakusan or keishibukuryogan. The data from the remaining 40 patients were used for external validation of the final model (Figure 1: the external validation set).

Table 2 summarizes the characteristics of the patients included in the two analyses. The frequency of OC use and diagnosed diseases were significantly higher in the external validation set than in the comparison and modeldevelopment set.

3.2. Comparison between the Characteristics of the TSS and $K B G$ Groups. We compared the characteristics of patients in the TSS group with those of patients in the KBG group (Table 3). The BMI was significantly lower in the TSS group; correspondingly, the binary variable "slim" was significantly more frequently present. Endometriosis or adenomyosis, which leads to secondary dysmenorrhea, was found in $13.3 \%$ of TSS patients and in $22.1 \%$ of KBG patients; however, this was not significantly different. The remainder of the patients in each group was diagnosed with primary dysmenorrhea.

Five subjective symptoms and three objective findings significantly differed between the TSS and KBG groups (Table 4; Appendix Table, at Supplementary Material available online at http://dx.doi.org/10.1155/2016/3159617). Lightheadedness was more frequent in patients in the TSS group; tendency to sweat, heat intolerance, leg numbness, and a cold sensation in the lower back were more frequent in patients in the KBG group. A weak abdomen was more frequent in the TSS group, whereas a strong abdomen, as well as paraumbilical tenderness and resistance, was more frequent in the KBG group. There was no significant difference between the two groups in terms of the other 123 subjective symptoms, seven abdominal findings, or two tongue findings.

3.3. A Predictive Model for Prescription of the 2 Kampo Formulas by Specialists. We performed univariate analyses of the five subjective symptoms and three abdominal findings that had shown a significant difference between the two groups, as well as of the variable "slim" (Table 5: univariate). We included a categorical variable "slim," rather than the continuous variable crude BMI, as linearity cannot be achieved on the logit scale when using crude BMI. We calculated AIC and AUC for each univariate model; all models had AIC > 150 and AUC $<0.8$ (Figure 2: univariate models).

We developed the full model using these nine potential predictive variables. The AIC for the full model was 127.9, and the AUC was 0.88 (95\% CI: 0.82-0.94, Figure 2: full model). The Hosmer-Lemeshow test indicated that the full model fitted the comparison and model-development set $(p=$ 0.1982).

After challenging all possible models, four subjective symptoms and three abdominal findings were included in the final model. The AIC for this model was 125.1, which was lower than that of the full model (Table 5: multivariate). None of the VIF values exceeded 2.0; thus, there was no collinearity in the model.

3.4. Internal and External Validations of the Final Model. The AUC was computed as 0.88 (95\% CI: 0.81-0.93, Figure 2: final model). The Hosmer-Lemeshow test indicated that the final model fit the comparison and model-development set 
TABLE 2: Baseline characteristics of the patients included in the study.

\begin{tabular}{|c|c|c|c|}
\hline & $\begin{array}{l}\text { Comparison and model- development set } \\
\text { May } 2008 \text { to March } 2013\end{array}$ & $\begin{array}{c}\text { External validation set } \\
\text { April } 2013 \text { to December } 2015 \\
\end{array}$ & $p$ value \\
\hline Number of patients & 128 & 40 & $\mathrm{~N} / \mathrm{A}$ \\
\hline \multicolumn{4}{|l|}{ Age at consultation } \\
\hline Mean \pm SD & $32.8 \pm 8.3$ & $35.0 \pm 7.0$ & $0.108^{*}$ \\
\hline Median & 33 & 37 & $0.129^{\dagger}$ \\
\hline Range & $12-50$ & $22-47$ & \\
\hline N/A & $0(0.0)$ & $0(0.0)$ & \\
\hline \multicolumn{4}{|c|}{ Age at menarche, years } \\
\hline Mean \pm SD & $12.3 \pm 1.5$ & $12.7 \pm 2.1$ & $0.245^{*}$ \\
\hline Median & 12 & 12 & $0.355^{\dagger}$ \\
\hline Range & $9-17$ & $10-21$ & \\
\hline N/A & $2(1.6)$ & $1(2.5)$ & \\
\hline \multicolumn{4}{|l|}{ Menstrual cycle, days } \\
\hline Mean \pm SD & $29.1 \pm 4.8$ & $28.2 \pm 2.6$ & $0.162^{*}$ \\
\hline Median & 28 & 28 & $0.076^{\dagger}$ \\
\hline Range & $16-60$ & $24-40$ & \\
\hline N/A & $22(17.2)$ & $9(22.5)$ & \\
\hline \multicolumn{4}{|l|}{ Bleeding period, days } \\
\hline Mean \pm SD & $5.9 \pm 1.6$ & $5.6 \pm 1.5$ & $0.366^{*}$ \\
\hline Median & 6 & 5 & $0.196^{\dagger}$ \\
\hline Range & $3-14$ & $3-10$ & \\
\hline N/A & $13(10.2)$ & $3(7.5)$ & \\
\hline \multicolumn{4}{|l|}{ BMI, kg/m² } \\
\hline Mean \pm SD & $20.8 \pm 3.1$ & $20.8 \pm 2.8$ & $0.905^{*}$ \\
\hline Median & 20.4 & 20.6 & $0.572^{\dagger}$ \\
\hline Range & $15.9-39.8$ & $16.2-31.6$ & \\
\hline$<18.5(\operatorname{slim})$ & $27(21.1)$ & $5(12.5)$ & $0.259^{\ddagger}$ \\
\hline$\geq 25$ (obese) & $11(8.6)$ & $2(5.0)$ & $0.735^{\ddagger}$ \\
\hline $\mathrm{N} / \mathrm{A}$ & $3(2.3)$ & $0(0.0)$ & \\
\hline \multicolumn{4}{|l|}{ Use of OCs } \\
\hline No & 117 (91.4) & $31(77.5)$ & $0.025^{\ddagger}$ \\
\hline Yes & $11(8.6)$ & $9(22.5)$ & \\
\hline \multicolumn{4}{|l|}{ Delivery } \\
\hline No & $116(90.6)$ & $35(87.5)$ & $0.556^{\ddagger}$ \\
\hline Yes & $12(9.4)$ & $5(12.5)$ & \\
\hline \multicolumn{4}{|l|}{ Abortion } \\
\hline No & $114(89.1)$ & $35(87.5)$ & $0.778^{\ddagger}$ \\
\hline Yes & $14(10.9)$ & $5(12.5)$ & \\
\hline \multicolumn{4}{|c|}{ Diagnosed organic disease } \\
\hline No & $105(82.0)$ & $25(62.5)$ & $0.016^{\ddagger}$ \\
\hline Endometriosis & $14(10.9)$ & $11(27.5)$ & $0.019^{\ddagger}$ \\
\hline Adenomyosis & $10(7.8)$ & $10(25.0)$ & $0.009^{*}$ \\
\hline \multicolumn{4}{|c|}{ Infertility (primary and secondary) } \\
\hline No & $123(96.1)$ & $45(87.5)$ & $0.146^{\ddagger}$ \\
\hline Yes & $5(3.9)$ & $5(12.5)$ & \\
\hline
\end{tabular}

N/A, not available; BMI, body mass index; OCs, oral contraceptives.

Findings are expressed as mean \pm SD, median, range, or number with percentage in parentheses.

$p$ values were calculated using ${ }^{*} t$-test, ${ }^{\dagger}$ Wilcoxon's rank sum test, and ${ }^{\ddagger}$ Fisher's exact test. 
TABLE 3: Baseline characteristics of the patients included in the comparison and model-development analysis.

\begin{tabular}{|c|c|c|c|c|}
\hline & \multicolumn{2}{|c|}{ The comparison and model development set } & \multirow{2}{*}{$p$ value } & \multirow{2}{*}{ Other formulas } \\
\hline & TSS group & KBG group & & \\
\hline Number of patients & 60 & 68 & $\mathrm{~N} / \mathrm{A}$ & 86 \\
\hline \multicolumn{5}{|l|}{ Age at consultation } \\
\hline Mean \pm SD & $33.3 \pm 7.9$ & $32.5 \pm 8.7$ & $0.595^{*}$ & $33.0 \pm 8.1$ \\
\hline Median & 33 & 33 & $0.742^{\dagger}$ & 33 \\
\hline Range & $17-50$ & $12-50$ & & $13-49$ \\
\hline $\mathrm{N} / \mathrm{A}$ & $0(0.0)$ & $0(0.0)$ & & $0(0.0)$ \\
\hline \multicolumn{5}{|c|}{ Age at menarche, years } \\
\hline Mean \pm SD & $12.2 \pm 1.5$ & $12.3 \pm 1.6$ & $0.753^{*}$ & $12.7 \pm 1.6$ \\
\hline Median & 12 & 12 & $0.932^{\dagger}$ & 12 \\
\hline Range & $9-16$ & $9-17$ & & $9-17$ \\
\hline $\mathrm{N} / \mathrm{A}$ & $0(0.0)$ & $2(2.9)$ & & $2(2.3)$ \\
\hline \multicolumn{5}{|l|}{ Menstrual cycle, days } \\
\hline Mean \pm SD & $29.0 \pm 5.7$ & $29.3 \pm 3.6$ & $0.772^{*}$ & $28.9 \pm 4.5$ \\
\hline Median & 28 & 28 & $0.771^{\dagger}$ & 28 \\
\hline Range & $16-60$ & $25-45$ & & $17-60$ \\
\hline N/A & $6(10.0)$ & $16(23.5)$ & & $10(11.6)$ \\
\hline \multicolumn{5}{|l|}{ Bleeding period, days } \\
\hline Mean \pm SD & $5.8 \pm 1.3$ & $5.9 \pm 1.9$ & $0.841^{*}$ & $5.5 \pm 1.6$ \\
\hline Median & 6 & 6 & $0.747^{\dagger}$ & 5 \\
\hline Range & $3-9$ & $3-14$ & & $3-14$ \\
\hline $\mathrm{N} / \mathrm{A}$ & $4(6.7)$ & $9(13.2)$ & & $7(8.1)$ \\
\hline \multicolumn{5}{|l|}{ BMI, $\mathrm{kg} / \mathrm{m}^{2}$} \\
\hline Mean \pm SD & $19.8 \pm 2.3$ & $21.6 \pm 3.5$ & $0.001^{*}$ & $20.6 \pm 2.9$ \\
\hline Median & 19.4 & 21.0 & $0.000^{\dagger}$ & 20.0 \\
\hline Range & $15.9-26.7$ & $17.0-39.8$ & & $15.6-30.1$ \\
\hline$<18.5(\operatorname{slim})$ & $21(35.0)$ & $6(8.8)$ & $0.000^{\ddagger}$ & $26(30.2)$ \\
\hline$\geq 25$ (obese) & $2(3.3)$ & $9(13.2)$ & $0.060^{\ddagger}$ & $7(8.1)$ \\
\hline N/A & $2(3.3)$ & $1(1.5)$ & & $4(4.7)$ \\
\hline \multicolumn{5}{|l|}{ Use of OCs } \\
\hline No & $54(90.0)$ & $63(92.6)$ & $0.754^{\ddagger}$ & $77(89.5)$ \\
\hline Yes & $6(10.0)$ & $5(7.4)$ & & $9(10.5)$ \\
\hline \multicolumn{5}{|l|}{ Delivery } \\
\hline No & $54(90.0)$ & $62(91.2)$ & $1^{\ddagger}$ & $75(87.2)$ \\
\hline Yes & $6(10.0)$ & $6(8.8)$ & & $11(12.8)$ \\
\hline \multicolumn{5}{|l|}{ Abortion } \\
\hline No & $53(88.3)$ & $61(89.7)$ & $1^{\ddagger}$ & $74(86.0)$ \\
\hline Yes & $7(11.7)$ & $7(10.3)$ & & $12(14.0)$ \\
\hline \multicolumn{5}{|c|}{ Diagnosed organic disease } \\
\hline No & $52(86.7)$ & $53(77.9)$ & $0.251^{\ddagger}$ & $81(94.2)$ \\
\hline Endometriosis & $6(10.0)$ & $8(11.8)$ & $0.785^{\ddagger}$ & $4(4.7)$ \\
\hline Adenomyosis & $2(3.3)$ & $8(11.8)$ & $0.103^{\ddagger}$ & $2(2.3)$ \\
\hline \multicolumn{5}{|c|}{ Infertility (primary and secondary) } \\
\hline No & $59(98.3)$ & $64(94.1)$ & $0.370^{\ddagger}$ & $81(94.2)$ \\
\hline Yes & $1(1.7)$ & $4(5.9)$ & & $5(5.8)$ \\
\hline
\end{tabular}

TSS, tokishakuyakusan; KBG, keishibukuryogan; N/A, not available; BMI, body mass index; OCs, oral contraceptives. Findings are expressed as mean $\pm S D$, median, range, or number with percentage in parentheses.

$p$ values were calculated using ${ }^{*} t$-test, ${ }^{\dagger}$ Wilcoxon's rank sum test, and ${ }^{\ddagger}$ Fisher's exact test. 
TABLE 4: Comparison of subjective symptoms and objective findings between the TSS and KBG groups.

\begin{tabular}{|c|c|c|c|}
\hline & The comparis & levelopment set & $p$ value \\
\hline & TSS group $(n=60)$ & KBG group $(n=68)$ & \\
\hline Subjectiv & & & \\
\hline Tender & & & \\
\hline No & $52(86.7)$ & $40(58.8)$ & 0001 \\
\hline Yes & $8(13.3)$ & $28(41.2)$ & 0.001 \\
\hline Heat in & & & \\
\hline No & $54(90.0)$ & $50(73.5)$ & 0.023 \\
\hline Yes & $6(10.0)$ & $18(26.5)$ & \\
\hline Leg nu & & & \\
\hline No & $59(98.3)$ & $59(86.8)$ & 0.019 \\
\hline Yes & $1(1.7)$ & $9(13.2)$ & 0.019 \\
\hline Cold sc & & & \\
\hline No & $57(95.0)$ & $56(82.4)$ & 0030 \\
\hline Yes & $3(5.0)$ & $12(17.6)$ & \\
\hline Lighth & & & \\
\hline No & $30(50.0)$ & $46(67.6)$ & 0049 \\
\hline Yes & $30(50.0)$ & $22(32.4)$ & 0.049 \\
\hline Objective & & & \\
\hline Weak a & & & \\
\hline No & $28(46.7)$ & $60(88.2)$ & 0.000 \\
\hline Yes & $32(53.3)$ & $8(11.8)$ & \\
\hline Strong & & & \\
\hline No & $58(96.7)$ & $50(73.5)$ & 0.003 \\
\hline Yes & $2(3.3)$ & $18(26.5)$ & 0.003 \\
\hline Paraun & & & \\
\hline No & $31(51.7)$ & $10(14.7)$ & 0.000 \\
\hline Yes & $29(48.3)$ & $58(85.3)$ & 0.000 \\
\hline
\end{tabular}

TSS, tokishakuyakusan; KBG, keishibukuryogan.

Only factors with $p$ value $<0.05$ were included. Findings are expressed as number with percentage in parentheses. $p$ values were calculated using Fisher's exact test.

TABLE 5: Effects of potential predictor variables and predictor variables in the final model.

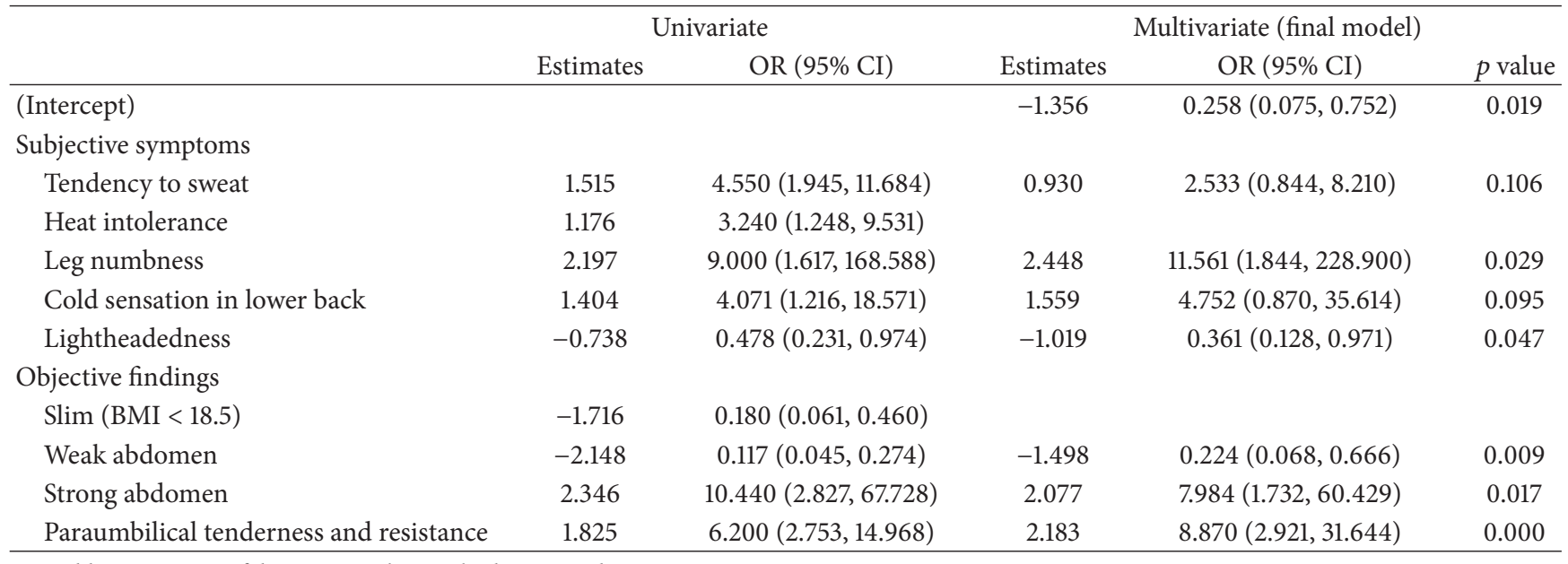

OR, odds ratio; CI, confidence interval; BMI, body mass index. 
TABLE 6: Internal and external validation of the final model.

\begin{tabular}{lc}
\hline & Accuracy (\%) \\
\hline Internal validation & \\
Internal estimate & 81.2 \\
Cross-validation estimate & 80.5 \\
External validation & 85.0 \\
\hline
\end{tabular}

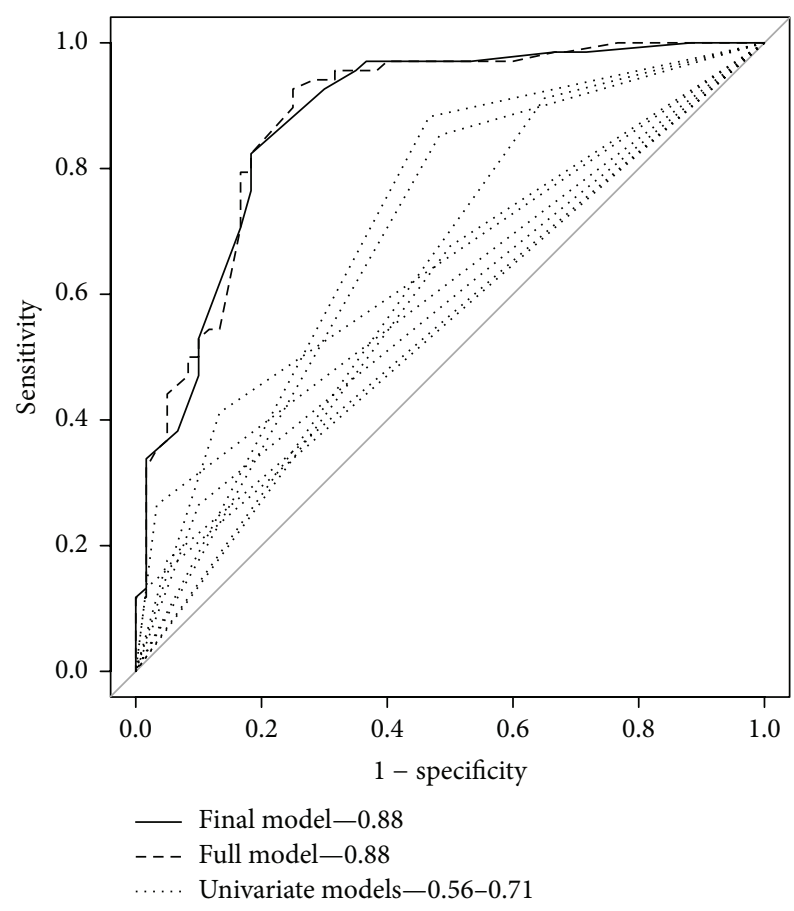

FIGURE 2: Model calibration using a receiver operating characteristic curve. The full model (broken line) included 9 predictive variables, and the final model (black line) included 7 predictive variables. Each univariate model was drawn using dotted lines. The final model had an area under the curve of 0.88 (95\% CI: 0.81-0.93), and the full model had an area under the curve of 0.88 (95\% CI: 0.82-0.94). The univariate models had areas under the curve of $0.56-0.71$ (95\% CI: not shown).

( $p=0.2519)$ better than did the full model. The internal estimate of accuracy of the final model was $81.2 \%$, and the leaveone-out cross-validation estimate of accuracy was $80.5 \%$ (Table 6: internal validation). When we applied this final model to the set of 40 external validation analysis patients, we found a proper prediction rate of $85.0 \%$ (Table 6 : external validation).

\section{Discussion}

Here, we have reported the differences in both subjective symptoms and objective findings between patients who had been prescribed tokishakuyakusan and those who had been prescribed keishibukuryogan. We extracted five subjective symptoms and four objective findings that were significantly different between these two groups. These items are compatible with the traditional medicine pattern diagnosis for each Kampo formula. Tokishakuyakusan is used for patients diagnosed with a "deficiency," "cold," "interior," "yin," "blood deficiency," and "fluid disturbance" pattern. From among these selected factors, a lower BMI and weak abdomen indicate a "deficiency" and a "yin" pattern. Lightheadedness indicates a "blood deficiency" or a "fluid disturbance" pattern. Conversely, keishibukuryogan is used for patients diagnosed with an "excess," "tangled heat and cold," "interior," "yang," and "blood stasis" pattern. Higher BMI and a strong abdomen indicate an "excess" and a "yang" pattern. A tendency to sweat, heat intolerance, and a cold sensation in the lower back indicate a "tangled heat and cold" pattern. Leg numbness, as well as paraumbilical tenderness and resistance, indicates a "blood stasis" pattern. Both formulas are used for an "interior" pattern; however, we found no item with $p<0.05$ that indicated an "interior" pattern.

Based on this differentiation, we have developed a predictive model, our final model, which fitted the data well. The final model quantified the tacit knowledge of Kampo specialists in selecting an appropriate Kampo formula for dysmenorrhea. During model selection, a subjective symptom-heat intolerance-and an objective finding-BMI-were eliminated from the final model, whereas all three abdominal findings were included in the final model. These results suggest that abdominal findings are important for specialists in selecting a Kampo treatment from among these two candidate formulas.

The selection of the appropriate formula is important in clinical situations. Each formula has specific characteristics and has been studied based on clinical experience. For example, tokishakuyakusan has been studied for its effect on infertility in rats and mice [23-26]. Keishibukuryogan has been studied for its effect on uterine myoma, not only in rats and mice, but also in humans [27-29].

Furthermore, the efficacy of each of these formulas is different from that of their individual crude constituents; thus, the combination of components is important [30]. For instance, tokishakuyakusan consists of six crude components: Japanese Angelica root, peony root, hoelen, Atractylodes rhizome, Alisma rhizome, and Cnidium rhizome. In contrast, keishibukuryogan consists of five crude components: cinnamon bark, peony root, hoelen, peach kernel, and moutan bark. Peony root is one of the crude drugs that tokishakuyakusan and keishibukuryogan have in common. A decoction of peony root has been used to treat many painful or inflammatory conditions, such as cholangitis, bronchiolitis, rhinorrhea, and muscle cramps. It has been reported to have an anticontraction effect, by suppressing the increase of intracellular calcium ion concentration, and anti-inflammatory effects, by inhibiting the production of prostaglandin E2, leukotriene B4, and nitric oxide [31]. However, some studies found that the isolated crude drug did not act as an anticontraction agent on uterine smooth muscle $[32,33]$.

The present study has some limitations. Our study involved many Kampo specialists, who may vary in their definitions of each finding. Such variations should be 
standardized with the advent of modern devices that can objectively examine a patient's tongue [34], abdominal wall [35], or pulse [36]. These objective findings will be incorporated into our model in the future to improve data reliability.

Second, clinical efficacy was not considered as part of this model development. More than $80 \%$ of our patients improved to at least some degree after Kampo treatment (data not shown), but retrospective validation of efficacy using medical charts was difficult and incomplete. Whether any formula is truly appropriate should be defined only by its carefully assessed efficacy. Moreover, we considered only the two representative Kampo formulas and did not consider other minor formulas. Although we performed a small external validation or our model, we excluded $41.2 \%$ of patients who were treated with minor formulas. If we apply our model in a clinical situation, approximately $40 \%$ of patients, who were treated with minor formulas, would have been prescribed either of the two major formulas. In the future, the effectiveness and safety of this model in a clinical situation should be evaluated using a prospective study design.

\section{Conclusions}

We compared the subjective symptoms and objective findings between patients who were prescribed either of the two major Kampo formulas used to treat dysmenorrhea (tokishakuyakusan and keishibukuryogan) and used this to develop a model that could predict the selection of either of these formulas for a patient by Kampo specialists. The effectiveness and safety of this model should be validated in prospective trials.

\section{Disclosure}

A part of this work was presented at the 33rd Annual Conference on Obstetrics and Gynecological Kampo Medicine Research.

\section{Conflict of Interests}

The authors declare that there is no conflict of interests regarding this work.

\section{Acknowledgments}

This work was supported by a Grant-in-Aid for Research on Propulsion Study of Clinical Research from the Ministry of Health, Labor, and Welfare. The authors would also like to thank Editage for providing editorial assistance.

\section{References}

[1] M. Proctor and C. Farquhar, "Diagnosis and management of dysmenorrhoea," The British Medical Journal, vol. 332, no. 7550, pp. 1134-1138, 2006.

[2] M. Y. Dawood, "Primary dysmenorrhea: advances in pathogenesis and management," Obstetrics and Gynecology, vol. 108, no. 2, pp. 428-441, 2006.
[3] A. S. Osayande and S. Mehulic, "Diagnosis and initial management of dysmenorrhea," American Family Physician, vol. 89, no. 5, pp. 341-346, 2014.

[4] Z. Harel, "Dysmenorrhea in adolescents and young adults: an update on pharmacological treatments and management strategies," Expert Opinion on Pharmacotherapy, vol. 13, no. 15, pp. 2157-2170, 2012.

[5] P. M. Latthe, R. Champaneria, and K. S. Khan, "Dysmenorrhoea," BMJ Clinical Evidence, vol. 2011, article 0813, 2011.

[6] M. L. Proctor and P. A. Murphy, "Herbal and dietary therapies for primary and secondary dysmenorrhoea," Cochrane Database of Systematic Reviews, no. 3, Article ID CD002124, 2001.

[7] A. Oya, T. Oikawa, A. Nakai, T. Takeshita, and T. Hanawa, "Clinical efficacy of Kampo medicine (Japanese traditional herbal medicine) in the treatment of primary dysmenorrhea," Journal of Obstetrics and Gynaecology Research, vol. 34, no. 5, pp. 898-908, 2008.

[8] J.-C. Pan, Y.-T. Tsai, J.-N. Lai, R.-C. Fang, and C.-H. Yeh, “The traditional Chinese medicine prescription pattern of patients with primary dysmenorrhea in Taiwan: a large-scale cross sectional survey," Journal of Ethnopharmacology, vol. 152, no. 2, pp. 314-319, 2014.

[9] W.-H. Sun, L. Zhao, X.-P. Tian, Q. Yang, and X. Li, “Clinical observation on treatment of 90 patients with primary menalgia with guizhi fuling capsule," Zhongguo Zhong Xi Yi Jie He Za Zhi Zhongguo Zhongxiyi Jiehe Zazhi, vol. 24, no. 12, pp. 1121-1123, 2004.

[10] N. Kotani, T. Oyama, I. Sakai et al., "Analgesic effect of a herbal medicine for treatment of primary dysmenorrhea-a doubleblind study," American Journal of Chinese Medicine, vol. 25, no. 2, pp. 205-212, 1997.

[11] World Health Organization, ICD-11 Beta Draft, WHO, 2015.

[12] X. Zhu, M. Proctor, A. Bensoussan, E. Wu, and C. A. Smith, "Chinese herbal medicine for primary dysmenorrhoea," Cochrane Database of Systematic Reviews, no. 2, Article ID CD005288, 2008.

[13] S. Yakubo, M. Ito, Y. Ueda et al., "Pattern classification in Kampo medicine," Evidence-Based Complementary and Alternative Medicine, vol. 2014, Article ID 535146, 5 pages, 2014.

[14] T. Yoshino, K. Katayama, K. Munakata et al., "Statistical analysis of Hie (cold sensation) and Hiesho (cold disorder) in Kampo Clinic," Evidence-Based Complementary and Alternative Medicine, vol. 2013, Article ID 398458, 8 pages, 2013.

[15] Y. Gepshtein, G. A. Plotnikoff, and K. Watanabe, "Kampo in women's health: Japan's traditional approach to premenstrual symptoms," Journal of Alternative and Complementary Medicine, vol. 14, no. 4, pp. 427-435, 2008.

[16] W. N. Venables and B. D. Ripley, Modern Applied Statistics with S, Springer, New York, NY, USA, 2002.

[17] H. Akaike, "Information theory and an extension of the maximum likelihood principle," in Proceedings of the 2nd International Symposium on Information Theory, B. N. Petrov and F. Caski, Eds., pp. 267-281, Akadimiai Kiado, Budapest, Hungary, 1973.

[18] D. W. Hosmer and S. Lemeshow, Applied Logistic Regression, John Wiley and Sons, New York, NY, USA, 2000.

[19] Team motRC, glm: Fitting Generalized Linear Models, 2015, https://stat.ethz.ch/R-manual/R-devel/library/stats/html/glm .html.

[20] J. H. Maindonald and W. J. Braun, DAAG: Data Analysis and Graphics Data and Functions, 2014, http://cran.r-project.org/ web/packages/DAAG/index.html. 
[21] X. Robin, N. Turck, A. Hainard et al., "pROC: Display and Analyze ROC Curves," 2015, https://cran.r-project.org/web/ packages/pROC/index.html.

[22] R. Subhash, J. L. K. Lele, and P. Solymos, "ResourceSelection: Resource Selection (Probability) Functions for Use-Availability Data," 2014, https://cran.r-project.org/web/packages/ResourceSelection/index.html.

[23] M. Irahara, T. Yasui, M. Tezuka et al., "Evidence that Tokishakuyaku-san and its ingredients enhance the secretion of a cytokine-induced neutrophil chemoattractant (CINC/gro) in the ovulatory process," Methods and Findings in Experimental and Clinical Pharmacology, vol. 22, no. 10, pp. 725-730, 2000.

[24] T. Koyama, N. Hagino, A. W. Cothron, and M. Saito, "Effect of Toki-Shakuyaku-San on ovulation induced by human menopausal gonadotropin in rats," American Journal of Chinese Medicine, vol. 16, no. 3-4, pp. 169-172, 1988.

[25] T. Koyama, N. Hagino, A. W. Cothron, and M. Saito, "Neuroendocrine effect of Toki-Shakuyaku-San on ovulation in rats," American Journal of Chinese Medicine, vol. 17, no. 1-2, pp. 29-33, 1989.

[26] H. Ota, S. Igarashi, and T. Tanaka, "Improved fertility through superoxide removal by Toki-shakuyaku-san in mice-a preliminary study," Reproduction, Fertility and Development, vol. 11, no. 7-8, pp. 451-455, 1999.

[27] T. Mori, S. Sakamoto, T. Singtripop et al., "Suppression of spontaneous development of uterine adenomyosis by a Chinese herbal medicine, Keishi-bukuryo-gan, in mice," Planta Medica, vol. 59, no. 4, pp. 308-311, 1993.

[28] S. Shinobu, K. Hideki, K. Tohru et al., "Effects of a Chinese herbal medicine, Keishi-bukuryogan, on the gonadal system of rats," Journal of Ethnopharmacology, vol. 23, no. 2-3, pp. 151-158, 1988.

[29] S. Sakamoto, H. Yoshino, Y. Shirahata, K. Shimodairo, and R. Okamoto, "Pharmacotherapeutic effects of kuei-chih-fu-lingwan (keishi-bukuryo-gan) on human uterine myomas," American Journal of Chinese Medicine, vol. 20, no. 3-4, pp. 313-317, 1992.

[30] M. Kushibiki, K. Yoshizaki, T. Tanaka, and M. Fukushima, "Study on the effect of Toki-shakuyaku-san extract and its serum in rats myometrial contraction," Journal of Traditional Medicines, vol. 17, no. 4, pp. 170-172, 2000.

[31] D.-Y. He and S.-M. Dai, "Anti-inflammatory and immunomodulatory effects of Paeonia lactiflora Pall., a traditional Chinese herbal medicine," Frontiers in Pharmacology, vol. 2, article 10, 2011.

[32] M. Harada, M. Suzuki, and Y. Ozaki, "Effect of Japanese Angelica root and Peony root on uterine contraction in the rabbit in situ," Journal of Pharmacobio-Dynamics, vol. 7, no. 5, pp. 304-311, 1984.

[33] G. Sumi, K. Yasuda, C. Kanamori et al., “Two-step inhibitory effect of kanzo on oxytocin-induced and prostaglandin F2 $\alpha$ induced uterine myometrial contractions," Journal of Natural Medicines, vol. 68, no. 3, pp. 550-560, 2014.

[34] T. Nakaguchi, K. Takeda, Y. Ishikawa et al., "Proposal for a new noncontact method for measuring tongue moisture to assist in tongue diagnosis and development of the tongue image analyzing system, which can separately record the gloss components of the tongue," BioMed Research International, vol. 2015, Article ID 249609, 10 pages, 2015.

[35] S. Yakubo, Y. Ueda, K. Muroga et al., "Modification of an abdominal diagnosis teaching simulator to reproduce patterns of resistance to pressure," Traditional and Kampo Medicine, vol. 2, no. 2, pp. 31-34, 2015.

[36] K. Y. Shin, S. O. Jin, S. H. Youn et al., "A pulse wave simulator for palpation in the oriental medicine," in Proceedings of the 33rd Annual International Conference of the IEEE Engineering in Medicine and Biology Society (EMBS '11), pp. 4163-4166, Boston, Mass, USA, September 2011. 


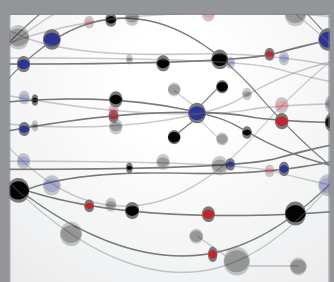

The Scientific World Journal
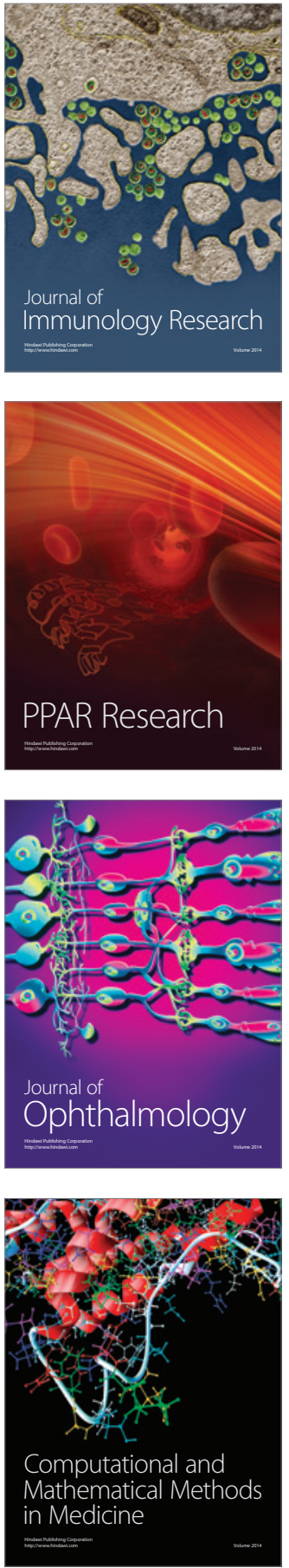

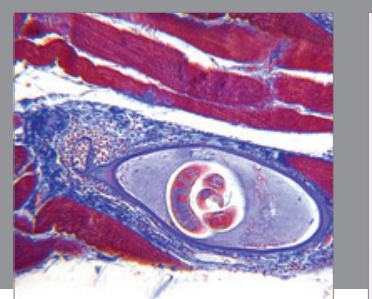

Gastroenterology Research and Practice

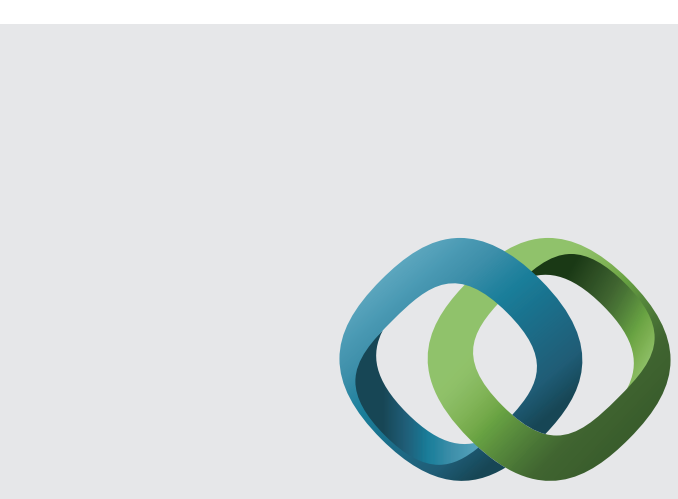

\section{Hindawi}

Submit your manuscripts at

http://www.hindawi.com
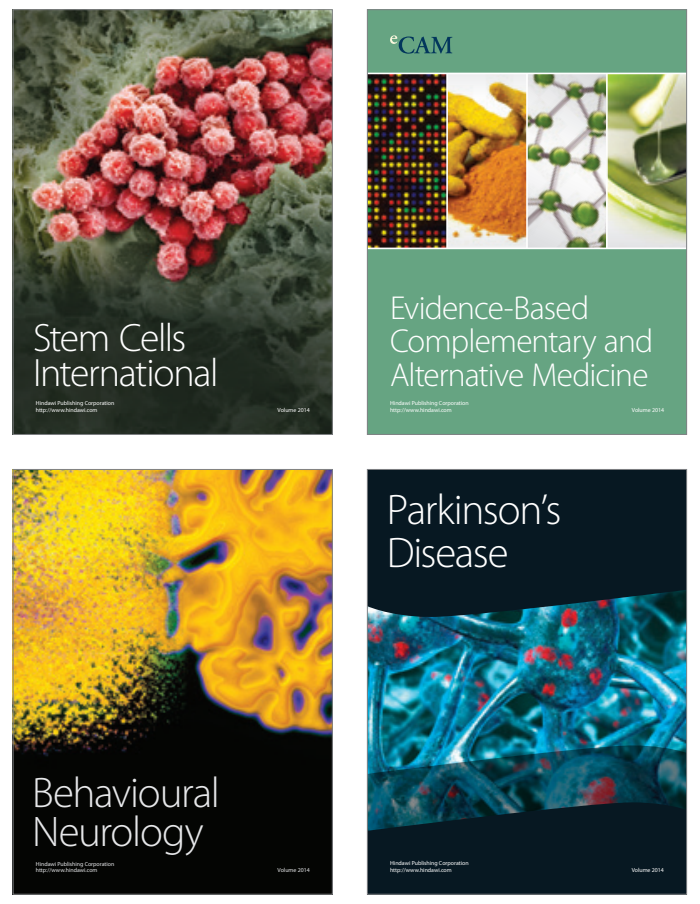
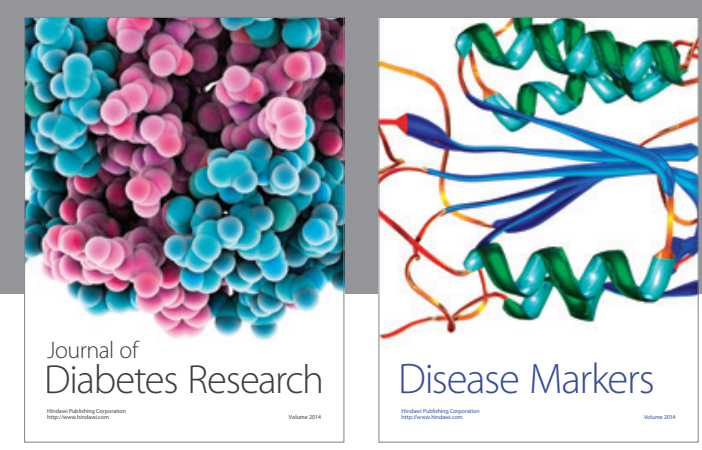

Disease Markers
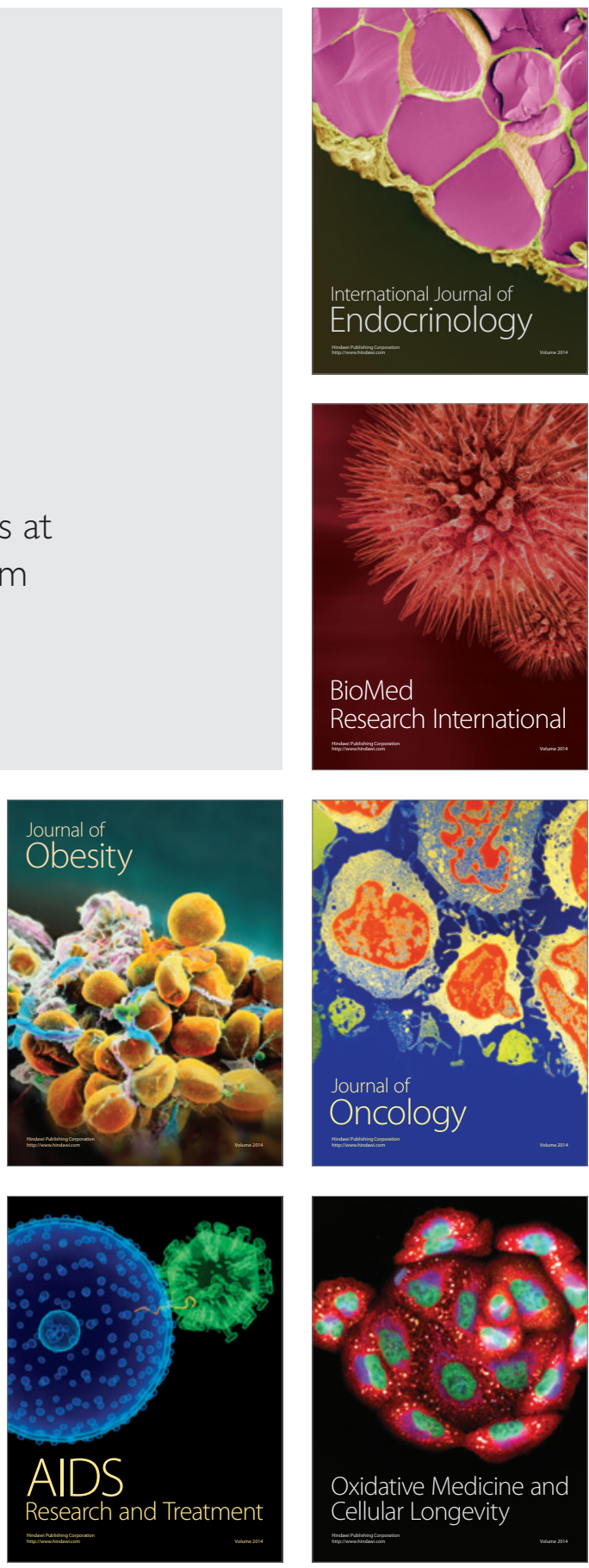